\title{
ESTRUTURA DE CAPITAL E JANELAS DE OPORTUNIDADE: TESTES NO MERCADO BRASILEIRO
}

\section{CAPITAL STRUCTURE AND WINDOWS OF OPPORTUNITIES: TESTS IN THE BRAZILIAN MARKET}

EDUARDO ALEXANDRE MENDES

Mestre em Administração de Empresas pelo Programa de Pós-Graduação em

Administração de Empresas da Universidade Presbiteriana Mackenzie (UPM). Departamento de Administração do Serviço Nacional de Aprendizagem Comercial (Senac). Rua Umbelino Bueno de Carvalho, 94 - São Paulo - SP - Brasil - CEP $01156-020$

E-mail: eamendes@gmail.com

\section{LEONARDO FERNANDO CRUZ BASSO}

PhD em Economia pelo Departmento de Economia da New School of Social Research, Estados Unidos.

Professor do Programa de Pós-Graduação em Administração de Empresas da Universidade Presbiteriana Mackenzie (UPM).

Rua da Consolação, 930, Consolação - São Paulo - SP - Brasil - CEP 01302-907

E-mail: leonardobasso@mackenzie.com.br

EDUARDO KAZUO KAYO

Livre-docente em Administração pela Faculdade de Economia, Administração e Contabilidade da Universidade de São Paulo (FEA-USP). Professor da Faculdade de Economia, Administração e Contabilidade da Universidade de São Paulo (FEA-USP). Avenida Professor Luciano Gualberto, 908 - São Paulo - SP - Brasil - CEP 05508-010 E-mail:kayo@usp.br 


\section{RESUMO}

Este estudo testa a teoria das janelas de oportunidade (EMTT) para o mercado acionário brasileiro. Este texto é uma adaptação do artigo de Baker e Wurgler (2002) que testaram com sucesso essa teoria para o mercado acionário americano. A teoria não foi comprovada no mercado brasileiro. A alavancagem diminuiu no primeiro ano após as ofertas públicas iniciais (IPO), mas cresceu novamente sem apresentar a persistência necessária para corroborar a teoria.

\section{PALAVRAS-CHAVE}

Oferta inicial de ações; Estrutura de capital; Janelas de oportunidade; Alavancagem; Criação de valor.

\section{ABSTRACT}

This study tests the market timing theory (EMTT) in the Brazilian Stock Market. The study is an adaptation from the Baker and Wurgler's article (2002) that successfully tested this theory in the American Stock Market. The theory has not been proven in the Brazilian Market. The leverage decreased in the first year after the initial public offerings (IPO), but it grew once again without presenting the persistency required to corroborate the theory.

\section{KEYWORDS}

Initial public offerings; Capital structure; Equity market timing; Leverage; Value Creation. 


\section{INTRODUÇÃO}

Desde as clássicas proposições de Modigliani e Miller (1958) sobre a irrelevância da estrutura de capital, inúmeras correntes teóricas sobre o tema têm surgido. A maioria delas contrapõe essas proposições, sugerindo que diversos fatores possam influenciar a estrutura de capital e o valor de uma empresa. Alguns dos fatores que determinariam a estrutura de capital seriam os benefícios fiscais, os custos de falência, os custos de agência, a lucratividade, a especificidade dos ativos, entre outros. Algumas correntes teóricas ganharam bastante destaque por suas contribuições nessa área. Segundo Harris e Raviv (I99I), quatro grandes correntes podem ser destacadas: a teoria de agência, a teoria de informações assimétricas, interações de mercado e controles corporativos (takeovers). Inúmeras pesquisas empíricas dentro dessas correntes já foram realizadas. Recentemente, uma abordagem empírica tendo por fundamento os princípios da teoria do equity market timing, ou das chamadas janelas de oportunidade, tem ganhado destaque.

Segundo Baker e Wurgler (2002), o equity market timing refere-se à prática de emitir ações a preços altos readquirindo-as a preços baixos. A teoria do equity market timing sugere que as empresas com maior alavancagem financeira são aquelas que captam recursos quando o valor de mercado da firma está em baixa. Por sua vez, as empresas com baixa alavancagem captam recursos externos quando o valor de mercado está em alta.

O objetivo deste artigo é testar a teoria do equity market timing para o mercado de ações brasileiro. Para tanto, procura-se analisar a influência dos valores de mercado históricos sobre a estrutura de capital das empresas brasileiras, tendo como base as datas de emissões públicas iniciais (IPO). A presente pesquisa é baseada na realizada por Baker e Wurgler (2002), mas apresenta algumas diferenças metodológicas importantes. Uma das diferenças mais importantes é o critério adotado, na presente pesquisa, para a definição da data da oferta pública inicial de ações (initial public offering - IPO). Baker e Wurgler (2002) assumem que a data da IPO das empresas analisadas coincide com a primeira aparição destas no banco de dados Compustat. Trata-se de uma premissa simplificadora, já que essa relação pode não ser necessariamente uma verdade. A presente pesquisa, de forma diferente, trabalha com as datas efetivas da oferta pública de ações. 


\section{REFERENCIAL TEÓRICO}

\subsection{AS TEORIAS DE ESTRUTURA DE CAPITAL}

A discussão atual sobre as questões que envolvem a estrutura de capital das empresas começa com as clássicas proposições de irrelevância de Modigliani e Miller (1958, I963). Segundo esses autores, não importa como uma empresa estrutura suas fontes de financiamento, pois o valor não se alteraria. A despeito dos inúmeros estudos já realizados, uma pergunta ainda persiste: existe uma estrutura ótima de capital? Em caso afirmativo, quais os determinantes dessa estrutura?

Muitas correntes teóricas contrariam a posição de Modigliani e Miller. Entre elas, três se destacam: a teoria do tradeoff, do pecking order e de agência. A teoria do tradeoff parte da premissa, de acordo com Myers (I984), que, mantidos constantes os ativos e os planos de investimento da empresa, o limite para o uso do capital de terceiros se dá quando os custos gerados pelo endividamento (que podem causar dificuldades financeiras) são maiores que os benefícios gerados pela economia fiscal.

A teoria do pecking order, por sua vez, admite que a estrutura de capital das empresas é baseada no conceito de hierarquização das fontes de financiamento, isto é, a premissa é de que as empresas prefiram ou priorizem o uso de uma fonte em relação a outra. De acordo com Myers (I984), as fontes de recurso podem ser obtidas de forma interna (fluxo de caixa da operação, retenção de dividendos etc.) ou externa (endividamento ou nova emissão de ações). Segundo Myers (I984), as empresas, em geral, preferem os financiamentos internos aos externos e o endividamento à nova emissão de ações. Segundo Myers (I984, p. 576) e Shyam-Sunder e Myers (I999, p. 220), a teoria do pecking order não prescreve um nível de endividamento "bem definido" ou ótimo. O endividamento se altera em função do desequilíbrio dos fluxos de caixa internos e das oportunidades de investimento. Mudanças no endividamento são direcionadas pela necessidade de recursos externos e não pela tentativa de se alcançar uma estrutura ótima de capital (SHYAM-SUNDER; MYERS, I999).

A teoria do pecking order é, aparentemente, uma das que mais se aderem ao mercado brasileiro. Gomes e Leal (2000), por exemplo, verificaram que a variável rentabilidade das empresas brasileiras analisadas é negativamente relacionada com o endividamento. Esses mesmos resultados também são encontrados por Nakamura (1992), Kayo (1997), Terra (2002), entre outros.

A teoria de agência se baseia no relacionamento entre principal e agente, pela qual o primeiro contrata o segundo para a execução de algum tipo de serviço 
(JENSEN; MECKLING, I976). Se cada um deles busca maximizar sua utilidade, as ações do agente nem sempre atendem aos interesses do principal, gerando então os conflitos de agência. De acordo com essa teoria, o endividamento ótimo é definido pela minimização do custo de agência total (soma do custo de agência do capital próprio externo e o custo de agência da dívida).

Uma das principais suposições da teoria da agência é a relação negativa entre oportunidades de crescimento e alavancagem financeira. Por exemplo, na fase de maturidade da empresa, quando as oportunidades de crescimento são pequenas, a utilização do capital de terceiros é predominante. Várias evidências empíricas corroboram essa tese, entre elas as de Kim e Sorensen (I986) e Lang, Ofek e Stulz (1996).

\subsection{MARKET TIMING E ESTRUTURA DE CAPITAL}

Korajczyk et al. (I990 apud HARRIS; RAVIV, I99I) afirmam que, tradicionalmente, o preço das ações tende a apresentar altas anormais em período antes da emissão de novas ações (IPO). Korajczyk et al. fizeram uma análise em crosssection entre a subida de preço e índices de endividamento relacionados ao $\mathrm{Q}$ de Tobin sobre o momento de emissão de ações. Esses autores descobriram que os índices de alavancagem financeira não aumentam anteriormente à emissão de ações, o que sugere que a capacidade de endividamento não é a principal causa na emissão de novas ações. O Q de Tobin (o índice de mercado em relação ao valor contábil dos ativos) é observado como sendo aumentado anteriormente à emissão de novas ações e cai após a emissão destas. Tais observações sugerem que a emissão de capital é utilizada para financiar novos investimentos.

Ritter (I99I) observou que, em longo prazo, o preço das ações se mostrou sobrevalorizado no momento de uma IPO. Essa constatação é de grande importância, pois traz relevantes constatações para investidores ao indicar que há uma certa precisão dos emissores ao aproveitar o que autor chama de "janela de oportunidades" (windows of opportunity) em momentos em que as ações estarão sobrevalorizadas, implicando aí custo de capital mais baixo para financiamento de novos investimentos.

Ainda segundo Ritter (I99I), empresas onde os ganhos no aftermarket são baixos tendem a apresentar um custo de capital próprio externo mais baixo. $\mathrm{O}$ autor estudou a relação entre performance aftermarket (após IPO) e outras variáveis, como tipo de indústria, porte da emissão, ano da IPO, idade da empresa etc. Tais estudos apontaram para uma forte evidência de que as empresas abrem o capital em momentos de auge no desempenho da indústria a qual pertencem, indicando haver uma certa dose de superestimação das oportunidades de crescimento em momentos de IPO por parte dos investidores. 
Além do desempenho da indústria (e outras variáveis microeconômicas), outros fatores podem atuar como importantes direcionadores de captação de recursos externos. Matsuo e Eid Jr. (2004) citam, por exemplo, o papel dos fatores macroeconômicos. Segundo esses autores, a correlação existente entre a taxa de juros e as emissões primárias é uma evidência do comportamento oportunista na escolha do momento mais adequado para a captação de recursos externos, caracterizando assim o market timing.

Outra importante evidência do oportunismo na captação de recursos no Brasil é apresentada por Eid Jr. (I996). Em uma pesquisa baseada em dados primários, levantados por questionário respondido por I6I empresas brasileiras, Eid Jr. (I996) mostra que a maioria das empresas pesquisadas (47\%) procura aproveitar as oportunidades oferecidas pelo mercado para a captação de recursos externos. Como destaca o autor, a teoria estática (tradeoff), que prevê um nível ótimo de endividamento em função do balanceamento entre os benefícios e custos das dívidas, pode não se aplicar ao mercado brasileiro.

Em outra pesquisa envolvendo questões macroeconômicas, Soares e Procianoy (2000) medem o impacto do Plano Real na questão da estrutura de capital das empresas brasileiras, para averiguar mudanças no perfil do endividamento. Para surpresa dos autores, houve poucas mudanças relacionadas tanto ao nível da alavancagem quanto ao perfil da dívida. Ou seja, mesmo com o fim da inflação, as empresas nacionais ainda encontram dificuldades de acesso ao crédito de longo prazo, resultando, dessa forma, numa menor alavancagem financeira se comparadas às empresas de outros países mais desenvolvidos, como observa Nakamura (I992).

A descoberta de que emissões de ações apresentam uma baixa performance na média implica custos mais baixos do que outras teorias sobre estrutura de capital previam em situações de capitação de capital próprio externo. Essa "janela de oportunidade" é apontada também por outros autores.

A nosso ver, este trabalho apresenta um importante apontamento no rol das teorias de estrutura de capital, pois oferece subsídios para futuros trabalhos que apontam para uma maior importância do mercado acionário na composição da estrutura de capital das empresas.

Loughran e Ritter (I997) demonstram diferenças de performance entre companhias que emitiram ações em um período de até 20 anos (entre i970 e i990) e companhias que não emitiram nesse período. As empresas que emitiram ações apresentaram uma performance inferior em relação àquelas que não realizaram alguma oferta pública no período estudado. Os autores consideraram tanto as ofertas públicas iniciais (IPO) quanto as ofertas de capital sazonais (SEO). Como principal resultado, constatou-se que os investidores que aportaram recursos em companhias emissoras tiveram que desembolsar $44 \%$ a mais em relação a inves- 
tidores que tivessem realizado aportes em companhias não emissoras para que seus retornos fossem equiparados.

Loughran e Ritter (I995) constataram ainda que a performance em situações de ofertas públicas varia em relação ao tempo. Empresas que realizaram emissões em períodos de baixo volume de emissões tiveram uma performance melhor do que aquelas que realizaram suas emissões em períodos de alta intensidade de emissões no mercado como um todo.

Esses autores constataram também que não há diferenças significativas em termos de performance entre ofertas iniciais (IPO) e ofertas sazonais (SEO). No entanto, a principal questão levantada foi explicar por que empresas que emitiram ações tiveram baixas performances nos cinco anos subsequentes à emissão.

Loughran e Ritter (I995) também constataram que a performance das empresas emissoras apresentou forte correlação negativa com a relação entre valor contábil e valor de mercado, ou seja, firmas com alto valor de mercado em relação ao valor contábil foram as que apresentaram as piores performances após os períodos de emissão.

Em suma, os autores concluíram como consistente a ideia de as empresas emitem ações em momentos em que os gestores as consideram, na média, sobrevalorizadas, sendo este momento denominado de "janela de oportunidades”. A principal explicação para esse fato não reside necessariamente na questão de assimetria de informação, mas em ineficiências de mercado relacionadas ao excesso de confiança dos investidores.

Partindo do pressuposto básico de que o mercado é ineficiente, Baker e Wurgler (2000) afirmam que as políticas de financiamento passam a ser relevante. Nesse caso, o preço do capital passa a ser a principal variável a influenciar na decisão de emitir novas ações, em que os acionistas são beneficiados em emissões nos momentos em que o preço das ações está baixo e são prejudicados em momentos em que o preço de mercado está subvalorizado.

Nesse estudo, Baker e Wurgler (2000) consideram as participações acionárias em novas emissões como um indicador mais consistente do que a distribuição de dividendos para medir a performance de um ano subsequente à emissão.

De acordo com essa hipótese, alguns autores como Ritter (I99I) e Loughran e Ritter (I995) confirmam a existência de uma baixa performance para as empresas emitentes logo após uma oferta pública inicial ou sazonal (IPO ou SEO). Essa baixa performance tem como principal importância para a teoria das finanças corporativas o fato de ir contra alguns conceitos defendidos em outras teorias clássicas, como as proposições básicas de Modigliani e Miller (i958), em que o mercado era tido como eficiente e a teoria pecking order de Myers (I984) ignorava alguns aspectos relacionados a oportunidades de mercado, invertendo assim a hierarquia de fontes de financiamento proposta por essa teoria. 
Existem varias formas para mensurar o equity market timing. Uma primeira medida para detectar o fenômeno está relacionada a retornos passados. Nesse enfoque, as firmas emitem ações quando os retornos das ações estão altos relativamente aos valores contábeis ou a valores de mercado passados, conforme estudo de Taggart (I977). Outra metodologia é apresentada por Korajczyk, Lucas e McDonald (I99I) que constataram que emissões de ações são mais prováveis de acontecer subsequentemente a anúncios de lucros positivos, uma medida que leva em conta o passado.

Um segundo enfoque para detectar ações sobrevalorizadas consiste em analisar a performance dos retornos subsequentes às emissões de ações.

Ritter (I99I) e Loughan e Ritter (I995) documentaram que emissões sazonais e IPO obtiveram desempenhos abaixo dos benchmarks no longo prazo.

O subdesempenho é mais pronunciado para hot market IPO (Ritter, I99I) e para IPO de firmas para as quais analistas previram altas taxas de crescimento (RAJAN; SERVAES, I997). Essas constatações dão suporte para enfoques que procuram diferenciar o momento pelo qual passa o mercado.

A conclusão é que os diretores financeiros (chief financial officers - CFO) admitem que explorar situações favoráveis do mercado de ações é um aspecto importante de suas decisões de financiamento.

Rajan e Zingales (I995) e Baker e Wurgler (2002) procuram capturar o fenômeno de market timing focando séries históricas da relação valor de mercado sobre o valor contábil.

Para averiguar quão persistente seria a influência do market-to-book, Baker e Wurgler (2002) criaram uma nova variável denominada média ponderada de financiamento externo do índice de mercado sobre valor contábil (external finance weighted-average, market-to-book ratio), representada doravante por $(M / B)_{\mathrm{efwa}, \mathrm{t}-\mathrm{I}}$, a qual foi calculada conforme a seguinte equação:

$$
\left(\frac{M}{B}\right)_{\text {efwa } t-1}=\sum_{s=0}^{t-1} \frac{e_{s}+d_{s}}{\sum_{r=0}^{t-1} e_{r}+d_{r}} \cdot\left(\frac{M}{B}\right)_{s}
$$

onde: $(e)$ e $(d)$ representam emissões de ações e de dívidas, respectivamente, e $(M / B)$ é o índice de valor de mercado sobre valor contábil já definido anteriormente.

Essa variável foi considerada, pois traz um efeito cumulativo de $(M / B)_{\mathrm{t}-\mathrm{I}}$, o que pode indicar uma relação de persistência dessa variável. Essa variável apresentou um coeficiente alto e negativo para empresas que aumentaram o nível de capital externo quando o índice de valor de mercado sobre valor contábil estava alto e vice-versa. 
Essa variável apresenta valores altos para empresas que contrataram recursos externamente quando a relação era alta e vice-versa. A motivação intuitiva para esse esquema de ponderação é que o financiamento externo representa oportunidades práticas para mudar a alavancagem: atribuir peso maior para avaliações que prevaleceram quando decisões significantes de financiamento externo estavam sendo feitas, não importando se essas decisões foram, no final das contas, pela opção do endividamento ou pela missão de ações.

Essa média ponderada é melhor que um conjunto de relações valor de mercado/valor contábil defasadas porque indica, para cada firma, precisamente que defasagens são as mais importantes.

Para propósitos de computar essa variável, eles fixaram o peso mínimo para zero e também excluíram observações anuais para as firmas onde o $\mathrm{M} / \mathrm{B}_{\text {efwa }}$ resultante excedeu a Io. O propósito de não permitir pesos negativos é assegurar a formação de uma média ponderada. Caso contrário, os pesos poderiam não estar aumentando no montante total de recursos contratados externamente em cada período, o que eliminaria a intuição que os pesos correspondem a períodos quando a estrutura de capital seria provavelmente mudada. Em todo caso, um peso zero significa que a variável não contém nenhuma informação sobre a avaliação de mercado naquele ano.

Existem outras possibilidades que são suportadas pela teoria; uma delas é regredir a variável dependente contra todos os market-to-book passados (desde o período (t-I) até o IPO, ou seja, uma série de market-to-book defasados).

Uma alternativa seria fazer uma ponderação que leva em consideração somente a emissão líquida de ações (e não uma emissão liquida de ações e dívidas). Baker e Wurgler (2002) testaram as alternativas e não encontraram discrepâncias entre os resultados.

Para prevenir uma conexão espúria devida à correlação entre a variável explicativa e as oportunidades de investimento, Baker e Wurgler (2002) excluem como variável explicativa a relação valor de mercado/valor contábil para o ano da IPO.

Alti (2003) questiona a metodologia de Baker e Wurgler argumentando que a relação valor de mercado/valor contábil é afetada pelo estado corrente da economia e pela intensidade de capital da tecnologia utilizada pela firma. Para caracterizar o estado da economia, Alti (2003) recupera e introduz os conceitos de mercado aquecido (hot market) e mercado desaquecido (cold market) conectados ao volume de IPO mensais. Mais especificamente, Alti (2003) determina o número de IPO para cada mês por um período de anos predeterminado (para amenizar variações sazonais, trabalha-se com uma média móvel de três meses para as IPO mensais). Meses aquecidos são definidos como aqueles que caem no mais alto quartil na distribuição da média móvel para todos os meses. Meses desaquecidos 
são aqueles que caem nos dois quartis inferiores; essa metodologia deixa margem para meses nem aquecidos nem desaquecidos, o que é justificado por Alti (2003) para criar uma separação incontestável entre os dois tipos de mercado. Deve-se enfatizar que esse enfoque não procura verificar se os market-timers são vitoriosos no longo prazo, ou seja, o enfoque procura identificar o fenômeno e não o sucesso ou fracasso dele. Pode acontecer que o desempenho superior esperado seja produto apenas da percepção dos emissores. A conclusão é que os emissores que emitem em mercados aquecidos emitem substancialmente mais ações que firmas que se tornam públicas quando o mercado não está aquecido. O efeito é robusto, pois não está associado a características atribuídas às firmas. Firmas que agem em mercados aquecidos apresentam um declínio considerável na alavancagem no ano da IPO. Entretanto, uma reversão na alavancagem se processa rapidamente. No ano seguinte ao da IPO, firmas que emitiram em mercados aquecidos emitem mais dívida de maneira perceptível, fazendo a alavancagem aumentar significativamente. Contrastando com esse comportamento, firmas que emitem em mercados desaquecidos se contentam em permanecer com a alavancagem do ano da IPO. Ao final do segundo ano, o efeito do mercado aquecido na alavancagem desaparece completamente. Alti (2003) conclui que o equity market timing é um determinante importante da atividade de financiamento no curto prazo, mas não no longo.

O equity market timing ainda não apresenta muitos testes no mercado brasileiro. Um deles é de autoria de Aggarwal, Leal e Hernandez (I993), que, a partir de uma amostra de 62 companhias brasileiras de capital aberto, constataram a existência de subdesempenho por volta de $47 \%$ em três anos após IPO, o que sugere sobrevalorização das ações brasileiras, corroborando os testes de Ritter (I99I), apesar de esses mesmos autores alertarem para o fato de tais conclusões serem tomadas com precaução por causa do reduzido tamanho da amostra utilizada e das concentrações de IPO em poucos anos. A pesquisa de Aggarwal, Leal e Hernandez (I993), entretanto, estuda a questão do market timing de uma outra perspectiva. O objetivo deles é verificar a influência da abertura de capital sobre o desempenho das ações e não sobre a estrutura de capital, que é o objetivo do presente artigo.

Um segundo artigo que analisa o market timing no Brasil é de autoria de Leite (2004). Esse autor também se baseia na metodologia de Baker e Wurgler (2002) para a realização de sua pesquisa. Entretanto, o critério de Leite (2004) para a definição da data de IPO difere do utilizado no presente artigo. É importante lembrar que, no presente artigo, se utiliza a data efetiva da IPO, ao passo que Leite (2004) utiliza um critério semelhante ao de Baker e Wurgler (2002), isto é, ele assume que a data da IPO corresponde ao primeiro ano em que a empresa aparece na base de dados utilizada, no seu caso a Economática. Independente- 
mente do critério utilizado, Leite (2004) também chega à mesma conclusão do presente artigo: que o valor de mercado não influencia a estrutura de capital. Em outra pesquisa, evidências apresentadas por Silva (2005) sugerem que o valor de mercado das ações apresenta uma relação negativa e significativa com o endividamento. A despeito das diferenças metodológicas entre essas pesquisas, as discrepâncias de resultado indicam a necessidade de novas investigações sobre o tema.

\section{METODOLOGIA}

\subsection{MÉTODO, PROBLEMA DE PESQUISA E OBJETIVOS}

O método aplicado nesta pesquisa é eminentemente quantitativo e descritivo. Com a investigação proposta, persegue-se o objetivo de se analisar a influência dos valores de mercado históricos sobre a estrutura de capital das empresas brasileiras, tendo as datas de emissões públicas iniciais (IPO) como pontos de referência. Esse objetivo implica testar se a teoria do equity market timing também se aplica ao mercado brasileiro. A suposição que fundamenta essa teoria é a de que as empresas emitem ações quando seu valor de mercado é alto e as recompram quando o valor é baixo. Além disso, pesquisas realizadas anteriormente mostram que, nos Estados Unidos, esse fenômeno é persistente por vários anos.

Isso posto, o problema de pesquisa que se pretende responder é: qual é a relação entre os valores de mercado históricos e a estrutura de capital das empresas brasileiras de capital aberto?

\subsection{PLANO AMOSTRAL E LEVANTAMENTO DOS DADOS}

A amostra analisada neste artigo foi escolhida por critério não probabilístico, e, portanto, os resultados não podem ser generalizados para a população. A amostra é composta por todas as empresas brasileiras não financeiras de capital aberto que realizaram operações de emissão pública inicial (IPO) entre I996 e 2002. A Figura I mostra o percentual de empresas analisadas por setor. Como se pode observar, existe a predominância de empresas do setor elétrico e de telecomunicações, fato justificável devido ao grande número de empresas que abriram capital na segunda metade dos anos I990, em razão do processo de privatizações. Apesar da predominância dos setores de energia e telecomunicações, há ainda uma representatividade diversificada de outros setores da economia. 


\section{FigurA I}

PERCENTUAL DE EMPRESAS ANALISADAS POR SETOR

Setores das Empresas Pesquisadas

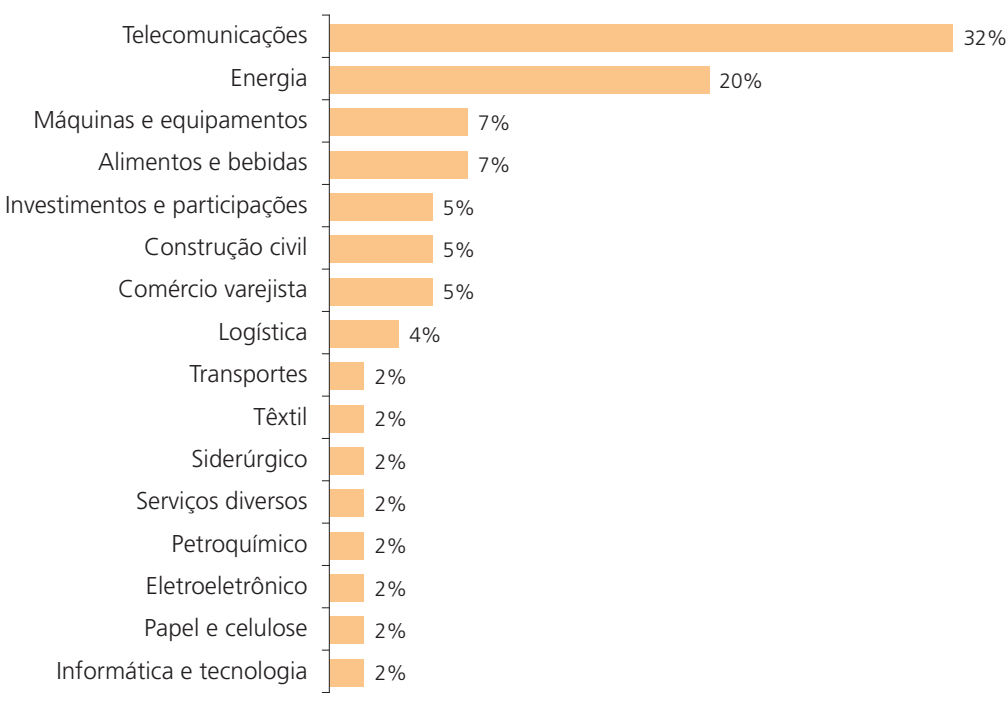

Fonte: Elaboração dos autores.

Algumas das análises processadas neste artigo testaram se a hipótese do equity market timing seria persistente ao longo de até cinco anos após o IPO. Para isso, foi necessário o levantamento dos dados econômico-financeiros de até cinco anos consecutivos para cada empresa. Dependendo do ano da IPO, evidentemente, uma empresa poderia não ter disponíveis os dados de todos esses anos. Se, por exemplo, uma empresa realizou o IPO no ano de 200I, seria possível realizar o teste apenas para o período IPO+I ano, já que o levantamento dos dados foi feito até o ano 2002. Para uma empresa que tenha feito a abertura de capital em 2000 , seria possível a realização da análise de até dois anos após o IPO e assim por diante. Por conta disso, a quantidade de casos analisados é decrescente na medida em que aumenta o período analisado. A Tabela I apresenta a quantidade de casos para cada um dos períodos analisados. Para o período IPO+I, por exemplo, a amostra inicial era composta por 6r empresas. Entretanto, em razão da falta de algum tipo de informação econômico-financeira, a amostra se reduz a 50 casos. Essa quantidade diminui na medida em que o período aumenta, até chegar a 27 empresas analisadas para o período IPO+5 anos. Por causa do reduzido número de empresas a partir do sexto ano, as análises ficaram restritas a cinco anos. 


\section{TABELA I}

AMOSTRA INICIAL E FINAL DE EMPRESAS

A PARTIR DO ANO DA IPO

\begin{tabular}{cccccc}
\hline Anos após a IPO & $\mathrm{IPO}+1$ & $\mathrm{IPO}+2$ & $\mathrm{IPO}+3$ & $\mathrm{IPO}+4$ & $\mathrm{IPO}+5$ \\
\hline Amostra inicial & 61 & 59 & 53 & 48 & 27 \\
\hline Amostra final & 50 & 49 & 44 & 38 & 27 \\
\hline
\end{tabular}

Fonte: Elaboração dos autores com base nos resultados da pesquisa.

O conhecimento da data da IPO permite examinar o comportamento da alavancagem a partir de um ponto de referência, nesse caso ao redor da data da oferta das ações, que é uma decisão de financiamento importante e se supõe estar relacionada à relação valor de mercado sobre valor contábil.

Este artigo é baseado no estudo de Baker e Wurgler (2002), mas a principal diferença entre aquele e este artigo é justamente a definição da data do IPO. Baker e Wurgler (2002) definem o ano de IPO como o primeiro ano no qual a Compustat informa dados de valor de mercado de uma empresa. Essa suposição pode levar a vieses no levantamento dos dados, uma vez que nada garante que a Compustat tenha incluído determinada empresa em sua base de dados, exatamente no ano que ela abriu seu capital. Para o presente artigo, o levantamento das datas de IPO foi baseado na data de registro da empresa na Comissão de Valores Mobiliários (CVM). Essa data de registro não difere muito da data de emissão das ações. A CVM, portanto, foi a primeira fonte de dados.

A segunda fonte de dados foi o banco de dados da Economática, de onde foram retirados os dados econômico-financeiros necessários para o cálculo das variáveis dependentes e independentes. Uma terceira fonte, a Lafis, também foi utilizada para o levantamento de alguns dados econômico-financeiros.

\subsection{OPERACIONALIZAÇÃO DAS VARIÁVEIS E TRATAMENTO DOS DADOS}

\subsubsection{Variáveis dependentes}

Todas as variáveis dependentes são, de alguma forma, relacionadas à estrutura de capital das empresas analisadas. No total, são seis as variáveis dependentes analisadas por meio de análises de regressão individuais. Quatro dessas variáveis se baseiam em variações entre um período e outro. As outras duas são variáveis correspondentes a um único período. A operacionalização é apresentada a seguir: 
- $\quad \Delta \mathrm{D} / \mathrm{A}_{\mathrm{t}}$ : variação na alavancagem financeira a valores contábeis entre as datas t e t-I, definida em termos percentuais como a razão entre o valor contábil do total das dívidas e obrigações sobre o total de ativos em valores contábeis.

- $\Delta \mathrm{e} / \mathrm{A}_{\mathrm{t}}$ : variação nas emissões líquidas de ações entre as datas t e t-I, definida como a variação do valor contábil do patrimônio liquido menos a variação no balancete de lucros retidos, sobre o total de ativos.

- $\quad \Delta \mathrm{RE} / \mathrm{A}_{\mathrm{t}}$ : variação nos lucros retidos recentes entre as datas t e t-I, definida como mudanças nos lucros retidos sobre o total de ativos.

- $\Delta \mathrm{d} / \mathrm{A}_{\mathrm{t}}$ : variação nas emissões liquidas de divida entre as datas te t-I, definida como a mudança residual em ativos sobre o total de ativos.

- $\mathrm{D} / \mathrm{A}_{\mathrm{t}}$ : alavancagem contábil no período $\mathrm{t}$, definida em termos percentuais como a razão entre o valor contábil das dívidas sobre o total de ativos.

- $\mathrm{D} /(\mathrm{A}-\mathrm{BE}+\mathrm{ME})$ : alavancagem financeira a valores de mercado no período $t$, definida em termos percentuais como a razão entre o valor contábil das dívidas (D) sobre o resultado da diferença entre o total de ativos (A) e o valor contábil do patrimônio líquido (BE) mais o valor de mercado das ações (ME), tanto ordinárias quanto preferenciais.

\subsubsection{Variáveis idependentes}

As principais variáveis independentes são relacionadas ao valor de mercado das empresas. São elas:

- $\quad \mathrm{M} / \mathrm{B}_{\mathrm{t}-\mathrm{I}}:$ a razão do valor de mercado sobre valor contábil na data t-I, onde $\mathrm{M}$ é igual ao total de ativos menos o valor contábil do patrimônio líquido mais o valor de mercado das ações, e B é o valor contábil do patrimônio líquido.

- $\quad \mathrm{M} / \mathrm{B}_{\text {efwa,t-I: }}$ razão valor de mercado sobre valor contábil, na data t-I, ponderada pelas emissões líquidas de dívidas e ações (que representam a fonte externa de financiamento).

Além das variáveis independentes principais, são incluídas no modelo algumas variáveis de controle que, segundo a literatura, seriam importantes determinantes da alavancagem financeira. São elas:

- $\quad$ PPE $/ \mathrm{A}_{\mathrm{t}-\mathrm{I}}$ : representa a tangibilidade dos ativos na data t-I e é medida por meio da razão entre o total do ativo imobilizado (PPE) sobre o total de ativos (A); a justificativa teórica para essa variável é a de que ativos fixos podem ser usados como garantia, diminuindo o risco do credor. Portanto, a relação entre a tangibilidade e a alavancagem seria positiva.

- $\quad$ EBTIDA/A $A_{t-I}:$ representa a lucratividade na data t-I e é medida por meio da razão entre os lucros antes de impostos, juros e depreciação (Ebtida) sobre 
o total de ativos (A). Segundo a teoria do pecking order, empresas mais lucrativas tenderiam a priorizar os recursos internos para financiar seus investimentos. Por esse ponto de vista, a relação entre alavancagem e lucratividade seria negativa.

- $\log (\mathrm{S})_{\mathrm{t}-\mathrm{I}}$ : representa o porte da firma na data t-I e é medido pelo logaritmo natural das receitas brutas $(\mathrm{S})$. O efeito do tamanho na alavancagem é ambíguo. Firmas maiores tendem a ser mais diversificadas e falham menos frequentemente. Assim, o tamanho pode ser considerado uma proxy inversa para a probabilidade de falência. Nessa interpretação, o tamanho apresenta um impacto positivo na oferta de dívida. No entanto, uma segunda corrente afirma que o tamanho é uma proxy para a informação que os investidores externos possuem, o que deveria aumentar sua preferência por ações, relativamente ao endividamento.

\subsubsection{Tratamento estatístico}

Os dados são analisados por meio da análise de regressão múltipla usando o método dos mínimos quadrados ordinários (MQO). Em uma primeira rodada de regressões, foi analisada a influência do valor de mercado do período anterior $\left(\mathrm{M} / \mathrm{B}_{\mathrm{t}-\mathrm{I}}\right)$, além das variáveis de controle, sobre as variações na estrutura de capital. Essas variações, como já explicado, são representadas por quatro variáveis dependentes. Cada variável dependente foi construída em diferentes períodos posteriores ao IPO. O termo IPO+I refere-se à variação da variável dependente entre o final do ano em que aconteceu o IPO e o primeiro ano posterior à emissão, IPO+2 é a variação entre o final do primeiro ano e o final do segundo ano, e assim por diante. O período de análise vai até o quinto ano seguido ao IPO. Portanto, cada variável dependente apresenta cinco regressões diferentes, cada uma correspondendo a um período.

Na segunda rodada de regressões, analisou-se a influência do valor de mercado e do valor de mercado ponderado, além das variáveis de controle, do período t-I sobre o endividamento do período $t$.

\section{RESULtAdOS DA PESQUISA}

\subsection{ESTATÍSTICA DESCRITIVA}

Os resultados da análise descritiva podem ser observados na tabelas 2 e 3 $\mathrm{Na}$ pesquisa para o Brasil, constatou-se comportamento semelhante ao apresentado por Baker e Wurgler (2002). Observa-se uma queda significativa na 
alavancagem contábil logo após a emissão primária. Como mostra a Tabela 2, a alavancagem contábil no período pré-IPO correspondia, em média, a 3I,o8\% do ativo total. No ano da IPO, essa média cai para $24,4 \%$ e se mantém abaixo da primeira alavancagem até o último ano analisado $(\mathrm{IPO}+5)$. Este seria um indício de que a teoria do market timing pode também ser aplicada à realidade brasileira, no entanto faz-se necessária a aplicação dos demais testes realizados por Baker e Wurgler (2002), conforme demonstrado mais adiante neste artigo.

\section{TABELA 2}

ESTATÍSTICAS DESCRITIVAS DAS VARIÁVEIS

DEPENDENTES DE ESTRUTURA DE CAPITAL

\begin{tabular}{|c|c|c|c|c|c|c|c|c|c|c|c|}
\hline \multirow[b]{2}{*}{ ANO } & \multirow[b]{2}{*}{$\mathrm{N}$} & \multicolumn{2}{|c|}{$\begin{array}{c}\text { ALAVANCAGEM } \\
\text { CONTÁBIL }\end{array}$} & \multicolumn{2}{|c|}{$\begin{array}{c}\text { ALAVANCAGEM } \\
\text { A VALORES DE } \\
\text { MERCADO }\end{array}$} & \multicolumn{2}{|c|}{$d / A_{t}$} & \multicolumn{2}{|c|}{$e / A_{t}$} & \multicolumn{2}{|c|}{$\triangle R E / A_{t}$} \\
\hline & & MÉD. & DP & MÉD. & DP & MÉD. & DP & MÉD. & DP & MÉD. & DP \\
\hline \multicolumn{12}{|c|}{ PAINEL A: TEMPO A PARTIR DA IPO } \\
\hline Pré-IPO & 41 & 31,08 & 21,02 & & & & & & & & \\
\hline IPO & 48 & 24,40 & 18,50 & 41,96 & 26,58 & 17,44 & 33,81 & 10,03 & 18,56 & 0,25 & 10,16 \\
\hline $\mathrm{IPO}+1$ & 50 & 26,80 & 20,28 & 40,29 & 25,19 & 11,46 & 18,00 & 8,45 & 31,60 & $-4,23$ & 19,53 \\
\hline $\mathrm{IPO}+2$ & 49 & 30,20 & 22,52 & 38,88 & 31,76 & 9,59 & 13,70 & 4,83 & 12,29 & $-3,02$ & 13,19 \\
\hline $\mathrm{IPO}+3$ & 44 & 29,64 & 16,98 & 43,82 & 21,99 & 2,17 & 7,90 & 0,32 & 7,29 & 4,75 & 12,38 \\
\hline $\mathrm{IPO}+4$ & 37 & 30,15 & 22,66 & 39,53 & 25,40 & 9,10 & 14,77 & 2,65 & 9,43 & $-3,21$ & 12,44 \\
\hline $\mathrm{IPO}+5$ & 27 & 25,65 & 21,45 & 40,91 & 25,46 & $-1,40$ & 16,03 & 8,00 & 4,45 & 2,16 & 5,81 \\
\hline \multicolumn{12}{|c|}{ PAINEL B: ANO-CALENDÁRIO (TODAS AS EMPRESAS) } \\
\hline 1997 & 33 & 20,58 & 17,88 & 64,50 & 31,84 & 21,17 & 27,00 & 42,12 & 31,64 & 14,05 & 27,44 \\
\hline 1998 & 42 & 21,63 & 17,35 & 46,63 & 22,94 & 30,06 & 39,18 & 10,64 & 20,87 & 4,34 & 8,71 \\
\hline 1999 & 50 & 23,20 & 19,75 & 35,45 & 25,80 & 18,04 & 26,51 & 5,26 & 9,78 & $-2,13$ & 6,15 \\
\hline 2000 & 49 & 28,96 & 17,68 & 42,02 & 23,76 & 8,76 & 20,49 & 10,74 & 33,59 & $-2,46$ & 18,78 \\
\hline 2001 & 50 & 31,63 & 19,59 & 39,43 & 25,45 & 9,44 & 18,15 & 3,50 & 12,25 & $-0,62$ & 10,40 \\
\hline 2002 & 47 & 31,98 & 26,38 & 33,24 & 34,75 & 11,01 & 16,36 & 3,30 & 11,62 & $-6,53$ & 15,03 \\
\hline
\end{tabular}

Fonte: Elaboração dos autores com base nos resultados da pesquisa. 
A Tabela 3 apresenta uma análise descritiva adicional do faturamento e do ativo total, da qual se pode observar o porte médio das empresas analisadas. Como é possível notar, as empresas analisadas podem ser consideradas de grande porte. Pelo painel A (tempo a partir da IPO), o faturamento médio é de, no mínimo, R\$ I.I38 milhão (na data do IPO), chegando a até R\$2.307 milhões (na data IPO+3). $\mathrm{O}$ ativo total, por sua vez, apresenta valores médios que vão de $\mathrm{R} \$ 2.070$ milhões (na data do IPO) a $\mathrm{R} \$ 2.824$ milhões (na data IPO+3).

\section{TABELA 3}

ESTATÍSTICAS DESCRITIVAS ADICIONAIS (R\$ MILHÕES)

\begin{tabular}{|c|c|c|c|c|c|}
\hline \multirow[b]{2}{*}{ ANO } & \multirow[b]{2}{*}{$\mathrm{N}$} & \multicolumn{2}{|c|}{ FATURAMENTO } & \multicolumn{2}{|c|}{ ATIVO TOTAL } \\
\hline & & MÉD. & DP & MÉD. & DP \\
\hline \multicolumn{6}{|c|}{ PAINEL A: TEMPO A PARTIR DA IPO } \\
\hline Pré-IPO & 41 & & & & \\
\hline IPO & 48 & 1.138 & 2.073 & 2.070 & 3.742 \\
\hline $\mathrm{IPO}+1$ & 50 & 1.472 & 2.787 & 2.360 & 4.510 \\
\hline $\mathrm{IPO}+2$ & 49 & 1.595 & 3.117 & 2.428 & 4.550 \\
\hline $\mathrm{IPO}+3$ & 44 & 2.307 & 3.806 & 2.824 & 5.216 \\
\hline $\mathrm{IPO}+4$ & 37 & 1.925 & 3.155 & 2.728 & 3.399 \\
\hline $\mathrm{IPO}+5$ & 27 & 1.862 & 2.180 & 2.669 & 3.347 \\
\hline \multicolumn{6}{|c|}{ PAINEL B: ANO-CALENDÁRIO (TODAS AS EMPRESAS) } \\
\hline 1997 & 33 & 417 & 415 & 737 & 1.257 \\
\hline 1998 & 42 & 718 & 1.258 & 1.740 & 2.738 \\
\hline 1999 & 50 & 1.076 & 1.679 & 2.029 & 3.338 \\
\hline 2000 & 49 & 1.471 & 2.541 & 2.279 & 3.719 \\
\hline 2001 & 50 & 1.822 & 3.022 & 2.789 & 4.705 \\
\hline 2002 & 47 & 2.018 & 3.327 & 3.040 & 4.922 \\
\hline
\end{tabular}

Fonte: Elaboração dos autores com base nos resultados da pesquisa. 


\subsection{DETERMINANTES DAS VARIAÇÕES ANUAIS NA ALAVANCAGEM}

Nessa primeira rodada de regressões, foi analisada a influência do valor de mercado da data $\mathrm{t}+\mathrm{I}\left(\mathrm{M} / \mathrm{B}_{\mathrm{t}-\mathrm{I}}\right)$ sobre as variações na estrutura de capital. Essas variações, como já explicado, são representadas por quatro variáveis dependentes. Além da variável explanatória principal, o valor de mercado, foram introduzidas ao modelo três variáveis de controle sugeridos pela literatura como determinantes da estrutura de capital. Assim, o modelo econométrico analisado é baseado na seguinte equação:

$$
\left(\frac{D}{A}\right)_{t}-\left(\frac{D}{A}\right)_{t-1}=a+b\left(\frac{M}{B}\right)_{t-1}+c\left(\frac{P P E}{A}\right)_{t-1}+d\left(\frac{E B T I D A}{A}\right)_{T-1}+e \log (S)_{t-1}+f\left(\frac{D}{A}\right)_{t-1}+u_{t}[2]
$$

A Tabela 3 apresenta os resultados da análise de regressão dos determinantes da variação da estrutura de capital. Essa tabela é dividida em quatro painéis. Cada painel compõe as regressões para cada uma das quatro variáveis dependentes. No painel A, por exemplo, apresentam-se as regressões tendo como variável dependente a variação na alavancagem contábil $\left(\Delta \mathrm{D} / \mathrm{A}_{\mathrm{t}}\right)$. Como se pode observar, não é possível afirmar que o valor de mercado influencie as variações na alavancagem contábil. O nível de significância é muito baixo nos cinco períodos analisados. Esse resultado não corrobora os estudos realizados por Baker e Wurgler (2002), que constataram relações negativas e significantes entre o valor de mercado e as variações na estrutura de capital.

\section{TABELA 4}

DETERMINANTES DAS VARIAÇÕES ANUAIS NA ALAVANCAGEM

\begin{tabular}{|c|c|c|c|c|c|c|c|c|c|c|}
\hline \multirow[b]{2}{*}{ ANO } & \multirow[b]{2}{*}{$\mathrm{N}$} & \multicolumn{2}{|c|}{$\log (S)_{t-1}$} & \multicolumn{2}{|c|}{$E B T I D A / A_{t-1}$} & \multicolumn{2}{|c|}{$P P E / A_{t-1}$} & \multicolumn{2}{|c|}{$\mathrm{M} / \mathrm{B}_{\mathrm{t}-1}$} & \multirow[b]{2}{*}{$R^{2}$} \\
\hline & & COEFIC. & SIGN. & COEFIC. & SIGN. & COEFIC. & SIGN. & COEFIC. & SIGN. & \\
\hline \multicolumn{11}{|c|}{ PAINEL A - VARIÁVEL DEPENDENTE: VARIAÇÃO NA ALAVANCAGEM CONTÁBIL } \\
\hline $\mathrm{IPO}+1$ & 50 & 0,01 & 0,85 & 0,04 & 0,27 & 0,08 & 1,19 & 0,00 & 0,77 & 0,06 \\
\hline $\mathrm{IPO}+2$ & 49 & 0,01 & 1,06 & $(0,19)$ & $(1,69)$ & 0,11 & 1,57 & 0,00 & 0,01 & 0,12 \\
\hline $\mathrm{IPO}+3$ & 44 & 0,01 & 1,55 & $(0,08)$ & $(0,66)$ & $(0,11)$ & $(2,00)$ & 0,05 & 2,23 & 0,24 \\
\hline $\mathrm{IPO}+4$ & 38 & 0,00 & 0,11 & 0,13 & 0,48 & 0,05 & 0,39 & $(0,10)$ & $(1,39)$ & 0,06 \\
\hline $\mathrm{IPO}+5$ & 27 & 0,00 & 0,44 & 0,06 & 0,56 & $(0,01)$ & $(0,19)$ & $(0,13)$ & $(2,43)$ & 0,27 \\
\hline
\end{tabular}




\section{TABELA 4 (CONTINUAÇÃO)}

\section{DETERMINANTES DAS VARIAÇÕES ANUAIS NA ALAVANCAGEM}

\begin{tabular}{|c|c|c|c|c|c|c|c|c|c|c|}
\hline \multirow[b]{2}{*}{ ANO } & \multirow[b]{2}{*}{ N } & \multicolumn{2}{|c|}{$\log (S)_{t-1}$} & \multicolumn{2}{|c|}{$\mathrm{EBTIDA} \mathrm{A}_{\mathrm{t}-1}$} & \multicolumn{2}{|c|}{$P P E / A_{t-1}$} & \multicolumn{2}{|c|}{$\mathrm{M} / \mathrm{B}_{\mathrm{t}-1}$} & \multirow[b]{2}{*}{$R^{2}$} \\
\hline & & COEFIC. & SIGN. & COEFIC. & SIGN. & COEFIC. & SIGN. & COEFIC. & SIGN. & \\
\hline \multicolumn{11}{|c|}{$\begin{array}{l}\text { PAINEL B - VARIÁVEL DEPENDENTE: VARIAÇÃO NA ALAVANCAGEM CONTÁBIL DEVIDA ÀS EMISSÕES } \\
\text { LÍQUIDAS DE AÇÕES }\end{array}$} \\
\hline IPO+1 & 50 & $(0,00)$ & $(0,42)$ & $(0,02)$ & $(0,15)$ & 0,00 & 0,06 & $(0,00)$ & $(12,72)$ & 0,89 \\
\hline $\mathrm{IPO}+2$ & 49 & 0,00 & 0,34 & 0,27 & 2,73 & $(0,08)$ & $(1,30)$ & $(0,06)$ & $(2,18)$ & 0,30 \\
\hline $\mathrm{IPO}+3$ & 44 & 0,01 & 1,96 & 0,05 & 0,50 & 0,05 & 1,13 & $(0,07)$ & $(3,26)$ & 0,29 \\
\hline $\mathrm{IPO}+4$ & 38 & $(0,01)$ & $(0,53)$ & 0,17 & 0,87 & $(0,02)$ & $(0,22)$ & $(0,01)$ & $(0,28)$ & 0,04 \\
\hline IPO+5 & 27 & $(0,00)$ & $(0,17)$ & $(0,08)$ & $(1,25)$ & 0,10 & 1,98 & $(0,02)$ & $(0,53)$ & 0,26 \\
\hline
\end{tabular}

PAINEL C - VARIÁVEL DEPENDENTE: VARIAÇÃO NA ALAVANCAGEM CONTÁBIL DEVIDA AOS LUCROS RETIDOS RECENTES

\begin{tabular}{|lc|cc|cc|cc|cc|c|}
\hline IPO+1 & 50 & $(0,00)$ & $(0,16)$ & $(0,03)$ & $(0,28)$ & $(0,02)$ & $(0,33)$ & 0,00 & 8,94 & 0,82 \\
\hline IPO+2 & 49 & 0,01 & 2,18 & $(0,52)$ & $(5,63)$ & $(0,11)$ & $(1,87)$ & 0,01 & 0,20 & 0,47 \\
\hline IPO+3 & 44 & $(0,00)$ & $(0,74)$ & $(0,39)$ & $(4,32)$ & $(0,04)$ & $(0,85)$ & 0,02 & 1,22 & 0,36 \\
\hline IPO+4 & 38 & 0,01 & 0,55 & $(0,48)$ & $(1,92)$ & $(0,03)$ & $(0,24)$ & $(0,04)$ & $(0,65)$ & 0,13 \\
\hline IPO+5 & 27 & 0,00 & 0,03 & $(0,02)$ & $(0,23)$ & $(0,01)$ & $(0,21)$ & $(0,07)$ & $(1,62)$ & 0,17 \\
\hline
\end{tabular}

PAINEL D - VARIÁVEL DEPENDENTE: VARIAÇÃO NA ALAVANCAGEM CONTÁBIL DEVIDA ÀS EMISSÕES LÍQUIDAS DE DÍVIDAS

\begin{tabular}{lc|cc|cc|cc|cc|c|}
\hline IPO+1 & 50 & & 1,36 & $(0,49)$ & $(2,62)$ & $(0,24)$ & $(2,71)$ & 0,00 & 0,45 & 0,25 \\
\hline IPO+2 & 49 & $(0,02)$ & $(2,21)$ & 0,22 & 1,79 & 0,06 & 0,73 & 0,02 & 0,44 & 0,11 \\
\hline IPO+3 & 44 & $(0,01)$ & $(0,88)$ & $(0,00)$ & $(0,00)$ & 0,01 & 0,13 & $(0,07)$ & $(1,91)$ & 0,13 \\
\hline IPO+4 & 38 & $(0,01)$ & $(0,40)$ & $(0,49)$ & $(1,63)$ & $(0,13)$ & $(1,02)$ & 0,04 & 0,54 & 0,12 \\
\hline IPO+5 & 27 & $(0,02)$ & $(0,64)$ & $(0,15)$ & $(0,59)$ & 0,22 & 1,14 & 0,05 & 0,40 & 0,09 \\
\hline
\end{tabular}

* Cada um dos painéis da Tabela 3 apresenta os resultados da análise de regressão tomando cada uma das diferentes variáveis dependentes. Em cada painel, são processadas cinco regressões diferentes: IPO+I refere-se à variação da variável dependente entre o final do ano em que aconteceu o IPO e o primeiro ano, IPO+2 é a variação entre o final do primeiro ano e o final do segundo ano, e assim por diante.

Fonte: Elaboração do autores com base nos resultados da pesquisa. 
A inclusão das variáveis de controle tampouco contribui para melhorar os resultados da regressão. Com raras exceções, as variáveis de controle não apresentam relação estatística significante.

\subsection{DETERMINANTES DA ALAVANCAGEM}

Na segunda rodada de regressões, foi analisada a influência dos determinantes da alavancagem, mas com a inclusão de mais uma variável, o valor de mercado ponderado pela emissão de dívidas e ações $\left(\mathrm{M} / \mathrm{B}_{\text {efwa,t-I }}\right)$. Assim, o modelo econométrico analisado é o seguinte:

$$
\left(\frac{D}{A}\right)_{t}=a+b\left(\frac{M}{B}\right)_{\text {effua },-1}+c\left(\frac{M}{B}\right)_{t-1}+d\left(\frac{P P E}{A}\right)_{t-1}+e\left(\frac{E B T I D A}{A}\right)_{t-1}+f \log (S)_{t-1}+u_{t}
$$

A Tabela 4 apresenta os resultados da análise de regressão dos determinantes da alavancagem. Vale lembrar que, nos estudos de Baker e Wurgler (2002), observou-se uma maior consistência, em termos de manutenção do $\mathrm{R}^{2}$ ao longo do tempo, para a variável $\mathrm{M} / \mathrm{B}_{\text {efwa,t-I }}$, o que indica alta correlação e persistência de valorações passadas de mercado em relação à estrutura de capital, como prediz a teoria do market timing. Entretanto, o mesmo não aconteceu para o Brasil. Como se pode observar, em todas as regressões, a variável $\mathrm{M} / \mathrm{B}_{\text {efwa,t-I }}$ apresentou relações negativas (embora nem sempre significantes) com as variáveis de alavancagem, como já era esperado. Entretanto, a variável M/Bt-I não apresentou os resultados previstos.

\section{TABELA 5}

\section{DETERMINANTES DA ALAVANCAGEM*}

\begin{tabular}{|c|c|c|c|c|c|c|c|c|c|c|c|c|}
\hline \multirow[b]{2}{*}{ Ano } & \multirow[b]{2}{*}{$\mathrm{N}$} & \multicolumn{2}{|c|}{$M / B_{\text {efwa, }} t-1$} & \multicolumn{2}{|c|}{$\mathrm{M} / \mathrm{B}_{\mathrm{t}-1}$} & \multicolumn{2}{|c|}{$P P E / A_{t-1}$} & \multicolumn{2}{|c|}{$\mathrm{EBTIDA} \mathrm{A}_{\mathrm{t}-1}$} & \multicolumn{2}{|c|}{$\log (S)_{t-1}$} & \multirow[b]{2}{*}{$R^{2}$} \\
\hline & & B & $T(b)$ & $b$ & $t(b)$ & $b$ & $t(b)$ & $b$ & $t(b)$ & $b$ & $t(b)$ & \\
\hline \multicolumn{13}{|c|}{ PAINEL A: ALAVANCAGEM CONTÁBIL EM \% } \\
\hline $\mathrm{IPO}+1$ & 50 & $(0,01)$ & $(0,26)$ & 0,01 & 0,26 & 0,03 & 0,34 & $(0,71)$ & $(3,30)$ & 0,03 & 2,36 & 0,28 \\
\hline $\mathrm{IPO}+2$ & 49 & $(0,06)$ & $(1,55)$ & $(0,01)$ & $(0,10)$ & 0,24 & 2,09 & $(0,65)$ & $(3,51)$ & 0,04 & 3,36 & 0,36 \\
\hline $\mathrm{IPO}+3$ & 44 & $(0,05)$ & $(1,94)$ & 0,08 & 1,75 & 0,25 & 2,52 & $(0,40)$ & $(1,80)$ & 0,03 & 2,91 & 0,38 \\
\hline $\mathrm{IPO}+4$ & 38 & $(0,10)$ & $(2,15)$ & 0,14 & 1,07 & 0,19 & 1,07 & $(0,81)$ & $(1,90)$ & 0,05 & 2,21 & 0,32 \\
\hline $\mathrm{IPO}+5$ & 27 & $(0,09)$ & $(4,82)$ & 0,23 & 3,16 & 0,21 & 1,96 & $(1,19)$ & $(8,69)$ & 0,02 & 1,35 & 0,86 \\
\hline
\end{tabular}




\section{TABELA 5 (CONTINUAÇ̃̃o)}

\section{DETERMINANTES DA ALAVANCAGEM*}

\begin{tabular}{|c|c|c|c|c|c|c|c|c|c|c|c|c|}
\hline \multirow[b]{2}{*}{ Ano } & \multirow[b]{2}{*}{ N } & \multicolumn{2}{|c|}{$M / B_{\text {efwa, }} t-1$} & \multicolumn{2}{|c|}{$M / B_{t-1}$} & \multicolumn{2}{|c|}{$P P E / A_{t-1}$} & \multicolumn{2}{|c|}{$\mathrm{EBTIDA} \mathrm{A}_{\mathrm{t}-1}$} & \multicolumn{2}{|c|}{$\log (S)_{t-1}$} & \multirow[b]{2}{*}{$R^{2}$} \\
\hline & & $B$ & $T(b)$ & $b$ & $t(b)$ & $b$ & $t(b)$ & $b$ & $t(b)$ & $b$ & $t(b)$ & \\
\hline \multicolumn{13}{|c|}{ Painel B: Alavancagem a valor de mercado em \% } \\
\hline $\mathrm{IPO}+1$ & 50 & $(0,03)$ & $(0,50)$ & 0,02 & 0,50 & 0,10 & 0,82 & $(0,99)$ & $(3,86)$ & 0,02 & 1,82 & 0,31 \\
\hline $\mathrm{IPO}+2$ & 49 & $(0,10)$ & $(2,89)$ & $(0,10)$ & $(1,67)$ & 0,36 & 3,29 & $(0,42)$ & $(2,39)$ & 0,02 & 2,36 & 0,46 \\
\hline $\mathrm{IPO}+3$ & 44 & $(0,13)$ & $(4,01)$ & 0,05 & 0,87 & 0,31 & 2,36 & $(0,41)$ & $(1,41)$ & 0,02 & 1,70 & 0,37 \\
\hline $\mathrm{IPO}+4$ & 38 & $(0,11)$ & $(3,19)$ & 0,02 & 0,18 & 0,13 & 0,96 & $(1,15)$ & $(3,53)$ & 0,05 & 2,83 & 0,52 \\
\hline $\mathrm{IPO}+5$ & 27 & $(0,13)$ & $(5,24)$ & 0,04 & 0,46 & 0,04 & 0,28 & $(0,94)$ & $(5,12)$ & 0,05 & 2,57 & 0,72 \\
\hline
\end{tabular}

*Cada um dos painéis da Tabela 4 apresenta os resultados da análise de regressão tomando cada uma das diferentes variáveis dependentes. Em cada painel, são processadas cinco regressões diferentes: IPO+I refere-se à variação da variável dependente entre o final do ano em que aconteceu o IPO e o primeiro ano, IPO+2 é a variação entre o final do primeiro ano e o final do segundo ano, e assim por diante.

Fonte: Elaboração dos autores com base nos resultados da pesquisa.

\section{CONSIDERAÇÕES FINAIS}

Para o Brasil, de modo geral, a replicação dos estudos realizados por Baker e Wurgler (2002) não obteve o mesmo resultado constatado para as empresas norte-americanas. As variáveis de controle, em algumas ocasiões, apresentaram as mesmas relações propostas pela literatura, embora muitas vezes sem significância estatística.

Vale ressaltar que a não comprovação das hipóteses de Baker e Wurgler (2002) para o Brasil pode ser devida às imensas diferenças encontradas em relação aos mercados de capitais dos dois países. A não comprovação de uma teoria que relaciona momentos de valoração de mercado com a composição da estrutura de capital das empresas pode ser explicada por diversos fatores conjunturais intrínsecos à realidade brasileira.

O fato de não se ter encontrado os resultados esperados, de acordo com o que prevê a literatura, pode parecer frustrante num primeiro momento. Entretanto, isso pode suscitar algumas reflexões sobre a realidade do mercado brasileiro. Em primeiro lugar, é notório o fato de que o mercado de capitais brasileiro é muito pouco desenvolvido em comparação ao norte-americano, por exemplo. No Brasil, 
a quantidade de empresas listadas em bolsa é reduzida e a liquidez das ações é baixa. Com isso, o preço das ações pode não refletir com confiabilidade o valor intrínseco das empresas.

Em segundo lugar, já que as ações podem não refletir o valor intrínseco, então o valor de mercado pode não ser um bom determinante da estrutura de capital no Brasil. Por isso, é primordial tentar entender, por meio de outras pesquisas que utilizem variáveis e teorias alternativas, as razões que levam à variação da estrutura de capital das empresas brasileiras.

\section{REFERÊNCIAS}

AGGARWAL, R.; LEAL, R. C.; HERNANDEZ, L. The aftermarket performance of initial public offerings in Latin America. Financial Management, p. 42-53, Spring I993.

ALTI, A. How persistent is the impact of market timing on capital structure? Austin: University of Texas, Oct. 2003 .

BAKER, M.; WURGLER, J. The equity share in new issues and aggregate stock returns. The Journal of Finance, v. 55, n. 5, p. 2219-2257, 2000.

Market timing e capital structure. The Journal of Finance, v. 57, n. I, p. I-32, 2002.

EID JR., William. Custo e estrutura de capital: o comportamento das empresas brasileiras. Revista de Administração de Empresas, São Paulo, v. 36, n. 4, p. 5I-59, out/nov/dez I996.

HARRIS, M.; RAVIV, A. The theory of capital structure. The Journal of Finance, v. 39, p. I27-I45, I99I.

JENSEN, M. C.; MECKLING, W. H. Theory of the firm: managerial behavior, agency costs and ownership structure. Journal of Financial Economics, v. 3, p. 305-360, 1976.

KAYO, E. K. Estrutura de capital e oportunidades de crescimento: uma análise dos determinantes do endividamento em diferentes contextos empresariais. 1997. Dissertação (Mestrado em Administração)-Faculdade de Economia, Administração e Contabilidade, Universidade de São Paulo, São Paulo, I997.

KIM, W. S.; SORENSEN, E. H. Evidence on the impact of the agency costs of debt in corporate debt policy. Journal of Financial and Quantitative Analysis, v. 2I, p. I3I-I44, I986.

KORAJCZYK, R.; LUCAS, D.; MCDONALD, R. The effects of information releases on the pricing and timing of equity issues. Review of Financial Studies, v. 4, p. 685-708, I99I.

Equity issues with timevarying asymmetric information. Journal of Financial \& Quantitative Analysis, v. 27, p. 397-417, 1992.

LANG, L.; OFEK, E.; STULZ, R. M. Leverage, investment, and firm growth. Journal of Financial Economics, v. 40, p. 3-29, I996.

LEAL, R. P. C.; DA COSTA JR., N. C. A.; LEMGRUBER, E. F. Finanças corporativas. São Paulo: Atlas: 200I. p. 42-57.

LEITE, E. C. Impacto das decisões de market timing nas companhias abertas brasileiras entre $1993 \mathrm{e}$ 2002. 2004. Dissertação (Mestrado em Administração)-Faculdade de Economia, Administração e Contabilidade, Universidade de São Paulo, São Paulo, 2004. 
LOUGHRAN, T.; RITTER, J. R. The new issues puzzle. The Journal of Finance, v. 50, p. 23-5I, I995.

. The operating performance of firms conducting seasoned equity offerings. Journal of Finance, v. 52, p. 1823-1850, 1997.

MATSUO, A. K.; EID JR., W. Influência de fatores macroeconômicos nas emissões primárias do mercado brasileiro. In: ENCONTRO BRASILEIRO DE FINANÇAS, Rio de Janeiro, 4., 2004.

MODIGLIANI, F.; MILLER, M. H. The cost of capital, corporation finance, and the theory of investment. American Economic Review, v. 48, p. 655-669, I958.

. Corporate income taxes and the cost of capital: a correction. American Economic Review,

v. 53 , p. $433-443,1963$.

MYERS, S.C. The capital structure puzzle. The Journal of Finance, v. 39, p. 575-592, I984.

NAKAMURA, W. T. Estrutura de capital das empresas no Brasil: evidências empíricas. I992. Dissertação (Mestrado em Administração)-Faculdade de Economia, Administração e Contabilidade, Universidade de São Paulo, São Paulo, I992.

RAJAN, R. G.; SERVAES, H. Analyst following of initial public offerings. Journal of Finance, v. 52, p. 507-529, I997.

RAJAN, R. G.; ZINGALES, L. What do we know about capital structure? Some evidence from international data. The Journal of Finance, v. 50, p. I42I-I460, I995.

RITTER, J. R. The long-run performance of initial public offerings. The Journal of Finance, v. 42, p. 365-394, I99I.

SHYAM-SUNDER, L.; MYERS, S. C. Testing static tradeoff against pecking order models of capital structure. Journal of Financial Economics, v. 51, n. 2, p. 219-244, Feb. I999.

SILVA, E. S. Desempenho das ações e a estrutura de capital das companhias abertas brasileiras nãofinanceiras. 2005. Dissertação (Mestrado em Administração)-Faculdade de Economia, Administração e Contabilidade, Universidade de São Paulo, São Paulo, 2005.

SOARES, K. T. C.; PROCIANOY, J. L. O perfil de endividamento das empresas negociadas em bolsa de valores de São Paulo após o Plano Real. In: XXIV ENCONTRO NACIONAL DA ANPAD, Florianópolis, 2000.

TAGGART, R. A. A model of corporate financing decisions. Journal of Finance, v. 32, I977.

TERRA, P. R. S. An empirical investigation on the determinants of capital structure in Latin America. In: ENANPAD - XXVI ENCONTRO NACIONAL DA ANPAD, Salvador, 2002. 